Is alcohol binge drinking in early and late pregnancy associated with behavioural and emotional development at age 7 years?

Niclasen, Janni; Andersen, Anne-Marie Nybo; Strandberg-Larsen, Katrine; Teasdale, Thomas William

Published in:

European Child \& Adolescent Psychiatry

DOI:

10.1007/s00787-013-0511-x

Publication date:

2014

Document version

Early version, also known as pre-print

Citation for published version (APA):

Niclasen, J., Andersen, A-M. N., Strandberg-Larsen, K., \& Teasdale, T. W. (2014). Is alcohol binge drinking in early and late pregnancy associated with behavioural and emotional development at age 7 years? European Child \& Adolescent Psychiatry, 23(12), 1175 - 1180. https://doi.org/10.1007/s00787-013-0511-x 


\title{
Is alcohol binge drinking in early and late pregnancy associated with behavioural and emotional development at age 7 years?
}

\author{
Janni Niclasen • Anne-Marie Nybo Andersen • \\ Katrine Strandberg-Larsen · Thomas William Teasdale
}

Received: 26 July 2013/ Accepted: 22 December 2013

(C) Springer-Verlag Berlin Heidelberg 2014

\begin{abstract}
The purpose of this study was to investigate associations of maternal binge drinking in early and late pregnancy with child behavioural and emotional development at age seven. It was hypothesised that late exposure is associated with more negative outcomes than early exposure. Differences were expected on the continuous outcome measures, but not on above cutoff scale scores. Data were derived from the Danish National Birth Cohort. Three exposure groups were defined according to binge drinking from three interviews regarding binge episodes in early, middle and late pregnancy. A 'no binge' group included women with no binge episodes reported in any of the interviews, the 'early bingers' reported episodes in the first interview only, and the 'late bingers' in the last part of pregnancy only. The outcome measure was the Strengths and Difficulties Questionnaire (SDQ) used as continuous externalising/internalising scores and above cutoff hyperactivity/inattention, conduct, emotional and peer problems scores. Only women with full information concerning binge drinking from the three interviews, together with full-scale SDQ information on their children at age seven and being term-born, were included in the study $(N=37,315)$. After
\end{abstract}

\footnotetext{
J. Niclasen $(\bowtie) \cdot$ T. W. Teasdale

Department of Psychology, University of Copenhagen, Øster Farimagsgade 2A, 1353 Copenhagen K, Denmark e-mail: janni.niclasen@psy.ku.dk

T. W. Teasdale

e-mail: tom.teasdale@psy.ku.dk

A.-M. N. Andersen - K. Strandberg-Larsen

Department of Public Health, Section of Social Medicine,

Øster Farimagsgade 5, 1014 Copenhagen K, Denmark

e-mail: amny@sund.ku.dk

K. Strandberg-Larsen

e-mail:ksla@sund.ku.dk
}

adjustment for maternal education, psychiatric diagnoses, age and smoking, children exposed to binge drinking in early and late pregnancy had significantly higher mean externalizing scores at age seven than unexposed children, an effect albeit much less for early binge drinking (relative change in mean 1.02, CI 1.00-1.05) than for late binge drinking (relative change in mean 1.21, CI 1.04-1.42). No associations were observed for any of the above cutoff outcomes. Exposure to binge drinking in early and late pregnancy is associated with elevated externalising scores, particularly so in late pregnancy. No increased risk for any of the above cutoff scale scores was observed.

Keywords Prenatal alcohol exposure $\cdot$ SDQ $\cdot$ Binge drinking · Danish National Birth Cohort - Timing of exposure $\cdot$ Strengths and difficulties questionnaire

\section{Introduction}

It is widely recognised today that prenatal exposure to alcohol, particularly at high levels, is negatively associated with neurobehavioural development [1]. It is also recognised that exposure to larger amounts of alcohol over a short period, for example in one evening, is more teratogenic to the developing central nervous system (CNS) than exposure to a comparable amount of alcohol spread over several days [2]. What is less known is the effect of timing, i.e. whether the developing CNS is particularly vulnerable to alcohol exposure in early and/or late pregnancy. Few human studies have investigated the importance of the timing of alcohol exposure during pregnancy on mental health outcomes in children [3-5] and no human studies have to our knowledge specifically investigated whether exposure to binge drinking, defined as sporadic high intake, 
in early and/or late pregnancy is associated with different mental health outcomes in childhood. This lack of evidence may be problematic as prenatal exposure to alcohol potentially interferes with all stages of brain development and different brain regions have unique courses of ontogeny [3, 4, 6-8]. Broadly speaking, the first trimester is mostly concerned with cell proliferation and migration [6], the second trimester with neuronal and synapse formation, axonal and dendritic outgrowth and programmed cell death [7], whereas in the third trimester the brain grows larger with synapse formation and myelination taking place [7].

Although it has been suggested that the timing of the binge exposure is very important [9], there is a general lack of human studies investigating the effects of timing on prenatal exposure to alcohol binge drinking. On the other hand, some animal studies have investigated such possible associations, and it has been suggested that what corresponds to the first and third trimesters in humans appears to be particularly sensitive periods for inducing CNS abnormalities [10]. One study [11] that examined the effects of gestational timing of alcohol exposure on neurobehavioural development in rhesus monkeys found that exposure to alcohol during early gestation significantly decreased scores on infant behavioural tests, whereas mid- to lategestation exposure resulted in reduced motor maturity, but did not affect overall neurobehavioural performance. According to the authors, this indicates that early-gestation alcohol exposure is as deleterious to neonatal neurobehaviour as late gestation or continuous exposure and that neurobehaviour is a more sensitive marker of early-gestation moderate alcohol exposure than growth parameters. Another study looking at rats [12] suggested that exposure during the equivalent of all three trimesters and third trimester equivalent significantly reduced Purkinje cell number compared with first and second trimester equivalent exposure, and that third trimester equivalent resulted in a decrement in the number of olfactory bulb mitral cells compared to first and second trimester equivalent exposure. Another rat study [13] investigated the effects of age, sex and timing of prenatal exposure to ethanol on social behaviour and inferred that early pregnancy exposure resulted in mild changes in social behaviour in young adolescents, whereas mid-gestation exposure resulted in pronounced behavioural deficits throughout ontogeny, with deficits being most robust in male offspring. Males exposed to ethanol in mid-gestation showed decreases in social investigation, contact behaviour and play fighting, whereas a decrease in social motivation was evident in adolescence regardless of sex. It was concluded that exposure to ethanol altered social behaviour and that the timing of the exposure defined the behavioural outcome.

Most previous studies based on human samples have defined prenatal exposure to alcohol solely from first trimester exposure. The aim of the present study was therefore to examine whether exposure to binge drinking in either early or late pregnancy was differently associated with behavioural and emotional development at age seven.

\section{Methods}

\section{Sample}

The data were derived from the population-based birth cohort, the Danish National Birth Cohort (DNBC) that comprises information on 100,418 pregnancies. The intention of the DNBC was to look at the association between exposures early in life and the health and development of the children from a longitudinal, life course perspective [14]. Between 1996 and 2002 pregnant women were enrolled nationwide at their first antenatal visit. The women were telephone interviewed twice during pregnancy at approximately weeks 16 and 30 and again 6 month postpartum. When the child reached 7 years of age, a questionnaire regarding the child's health and development was sent to the mother. All questionnaires are available in English at http://www.dnbc.dk [15].

\section{Restriction of sample}

The sample was restricted to mothers who answered all questions regarding binge drinking in the first three interviews, who completed the Strengths and Difficulties Questionnaire (SDQ) at age seven of the child and for whom the child was a singleton with a gestational age of $\geq 37$ completed weeks. These restrictions resulted in a total of 37,315 mother-child dyads. The mothers were informed that the data were being collected for research purposes and gave oral consent to this. They were informed that statistical results would be reported in such a way that individuals could not be identified. Ethical approval was obtained for the study.

Of the total 37,315 mothers, 25,781 reported no binge drinking episodes during pregnancy and constituted a 'no binge' control group. The early exposure group consisted of women reporting binge drinking in the first interview, but no binge episodes in the second and third interviews $(N=3,654)$. The late exposure group consisted of women reporting binge episodes in the third interview, but no binge episodes in the first and second interviews $(N=94)$. The remaining exposure groups were excluded from all analyses. The excluded groups included: reported binge episode(s) in the second interview only $(N=1,990)$, binge episodes according to both the first and third interviews $(N=48)$, binge episodes according to all three interviews $(N=155)$, binge episodes in the first and second 
interviews $(N=5,535)$ and binge episodes in the second and third interviews $(N=58)$. The binge groups were defined on the basis of exposure to binge drinking only, i.e. not including information on cumulated alcohol exposure.

Exposure: alcohol binge drinking

A standard drink in Denmark is defined by the National Board of Health as $12 \mathrm{~g}$ of absolute alcohol. Binge drinking was defined as an intake of five or more alcohol-containing units on a single occasion. In the first interview the women were asked: "If you think about the entire period of pregnancy, also the very beginning, how many times did you then have five drinks or more in one night/event"? If a woman answered yes to this question she was further asked about "the number times" and at "what week(s) of gestation she was in the 1 st, 2nd, 3rd, etc. times". The phrasing of the binge drinking questions was similar in the second interview, i.e. enquiring about the number of episodes from the beginning of pregnancy. In the third interview the women were asked about binge episodes from week 30 and until birth. Binge drinking was assessed separately on the basis of information from three interviews conducted approximately in week 16 regarding early pregnancy intake, approximately in week 30 regarding middle pregnancy intake and 6 months postpartum concerning intake in the last part of pregnancy. If a woman reported any binge episodes in the first interview and none later, she was considered an 'early' pregnancy binger, if she reported any such episodes in the second interview she was considered a middle pregnancy binge drinker (and therefore excluded) and if she reported occasions of binge drinking during pregnancy in the third interview with none earlier she was considered a late pregnancy binge drinker. All women reporting any occasions of binge drinking were further asked about the total number and pregnancy weeks of each binge episode. The present study is concerned with women who reported binge drinking exclusively early or exclusively late in pregnancy as defined here. A control group was included comprising women who reported never binge drinking during pregnancy.

\section{Outcome: parent-rated SDQ}

The SDQ contains 25 items concerned with five domains of psychological adjustment: hyperactivity/inattention (hereafter hyperactivity), conduct, emotional, peer problems and prosocial behaviours. Each item is scored on a three-point Likert scale; 'not true', 'somewhat true' and 'certainly true' yielding scores between 0 and 2 for each question [16-18]. Because the aim was to identify problem behaviours, the prosocial scale was not used in the present study. The four problem scales were used both as four separate scale models (i.e. hyperactivity, conduct, emotional and peer problems) and in a broader model of externalising (combining the hyperactivity and conduct scales) and internalising scales (combining the emotional and peer problem scales) as these two models have been found to have equally good statistical fits [19]. The externalising and internalising scales were used as continuous outcome variables and the four problem scales as dichotomised clinical cutoffs (approximately, the highest scoring $20 \%$ ). The following cutoffs were adopted: hyperactivity ( $\geq 5$ for boys and $\geq 4$ for girls), emotional ( $\geq 4$ for boys and girls), peer problems ( $\geq 2$ for boys and girls) and conduct problems scores ( $\geq 3$ for boys and girls) [20].

\section{Confounders}

The following potential confounding factors were controlled for: maternal education (9 years or less, 10-12 years, 13 years or more); maternal psychiatric diagnosis up to the age of seven of the child (yes/no), maternal age and maternal smoking in pregnancy (yes/no). Information on past psychiatric history, education and age came from registries, and the smoking variable from selfreports. Because only women who have been in contact with the psychiatric services are recorded in the Danish psychiatric central registry and, because it is the vulnerability of the women that is considered a confounder, all women who had been in contact with the psychiatric system up to the age of seven of their child were included as cases in the psychiatric diagnoses variable.

\section{Statistical analyses}

All analyses were carried out in SAS version 9.2. The primary aims were to investigate: (1) associations between exposure to binge drinking in early and late pregnancy and continuous internalising/externalising SDQ scores at age seven; (2) associations between binge drinking in early and late pregnancy and above cutoff hyperactivity, conduct, emotional and peer problems scores at age seven. The secondary aim was to perform two sets of sensitivity analyses to test the effect of the number of binge episodes and the stability of the result after restricting the early binge group to women interviewed before 16 completed weeks of gestation. Multivariable linear regressions were used to model binge drinking and associations with continuous externalising/internalising SDQ scores. Because the distribution of SDQ scores have been found to be positively skewed [21], the internalising/externalising scale scores were log transformed before use as continuous variables in the multivariable linear regressions and the outcomes therefore reflect relative changes in mean. The four problem scales (hyperactivity, conduct problems, peer 
problems and emotional symptoms) were used in logistic regression models with appropriate cutoffs identifying the $20 \%$ of the sample with the highest problems scores.

In the main analyses all women, regardless of the number of binge episodes, were included. To test the robustness of the results the analyses were rerun including women with one or two binge episodes only, i.e. excluding women with three or more episodes. Further, all analyses were rerun including only women who were interviewed before 16 completed gestational weeks in the early exposure group, to increase the reliability of the early binge drinking reports.

\section{Results}

Table 1 shows the background characteristics of the three exposure groups. ANOVA and Chi square probability testing were performed to compare the 'no binge' group with the 'early' and 'late' binge groups. It appears that both binge exposure groups had higher percentages of women living in rented accommodation (29.3 and $29.8 \%$ ), but probably because of the small size of the late binge group only the early binge group differed significantly from the no binge group. Both exposure groups differed significantly from the no binge group on the maternal education variable, although in opposite directions. Only $38.3 \%$ of the late binge drinkers had 13 years or more of education compared to $52.3 \%$ of the non-bingers. It is also apparent from Table 1 that $10.6 \%$ of the mothers in the late binge group had had contact with the psychiatric system up to the age of seven of the child. This is twice as many as in the other groups and significantly different from the no bingers. Interestingly, the opposite is true for the fathers of the late binge group where only $1.1 \%$ have had contact with the psychiatric system before the age of seven of the child. As was expected, significantly more early and late binge drinking mothers smoked compared to the non-binge group and the two binge exposure groups also had a lower proportion of planned pregnancies.

Tables 2 and 3 present the adjusted relative changes in means for the continuous externalising and internalising scores and number of cases as well as odds ratios (OR) for the dichotomised measures on the hyperactivity, conduct, peer problem and emotional scales, respectively. The models were initially controlled for maternal education, psychiatric diagnoses, age, cumulated alcohol intake in pregnancy and smoking in pregnancy. However, as the cumulated alcohol variable did not contribute significantly to the model, it was removed and thus not controlled for in any of the analyses. Table 2 shows that externalising scores are significantly associated with binge drinking in early [relative change in mean 1.02 (CI 1.00-1.05)] and late
Table 1 Background characteristics of the three exposure groups

\begin{tabular}{|c|c|c|c|}
\hline & No binge & Early binge & Late binge \\
\hline \multirow[t]{2}{*}{$N$} & 25,781 & 3,654 & 94 \\
\hline & $\begin{array}{r}\text { Means } \\
(\mathrm{SD})\end{array}$ & Means (SD) & Means (SD) \\
\hline Maternal age & $30.8(4.2)$ & $\begin{array}{l}30.4 \\
\quad(4.2) * * * *\end{array}$ & $31.0(4.5)$ \\
\hline Paternal age & $32.6(5.1)$ & $\begin{array}{l}32.1 \\
\quad(5.2) * * * *\end{array}$ & $32.6(6.0)$ \\
\hline \multirow[t]{2}{*}{$\begin{array}{l}\text { Cumulated alcohol } \\
\text { intake in pregnancy }\end{array}$} & $26.7(38.4)$ & $\begin{array}{l}42.0 \\
\quad(51.3) * * * *\end{array}$ & $\begin{array}{l}64.1 \\
\quad(63.7) * * * *\end{array}$ \\
\hline & Percentages & Percentages & Percentages \\
\hline $\begin{array}{l}\text { In rented } \\
\text { accommodation (yes) }\end{array}$ & 23.8 & $29.3 * * * *$ & 29.8 \\
\hline \multicolumn{4}{|l|}{ Maternal education } \\
\hline $0-9$ years of education & 7.0 & 5.9 & 7.5 \\
\hline $\begin{array}{l}\text { 10-12 years of } \\
\text { education }\end{array}$ & 40.7 & 39.8 & 54.3 \\
\hline$>13$ of education & 52.3 & $54.2 *$ & $38.3 *$ \\
\hline \multicolumn{4}{|l|}{ Paternal education } \\
\hline $0-9$ years of education & 12.4 & 11.5 & 18.1 \\
\hline $\begin{array}{l}\text { 10-12 years of } \\
\text { education }\end{array}$ & 47.2 & 47.4 & 43.6 \\
\hline$>13$ years of education & 40.4 & 41.1 & 38.3 \\
\hline $\begin{array}{l}\text { Maternal psychiatric } \\
\text { diagnosis }\end{array}$ & 4.8 & 5.2 & $10.6^{* *}$ \\
\hline $\begin{array}{l}\text { Paternal psychiatric } \\
\text { diagnosis }\end{array}$ & 3.4 & 3.7 & 1.1 \\
\hline Maternal smoking & 18.91 & $30.62 * * * *$ & $32.26 * * *$ \\
\hline Paternal smoking & 26.2 & $30.5 * * * *$ & 27.7 \\
\hline $\mathrm{BMI}^{\mathrm{d}}$ & 68.81 & $72.15^{* * * *}$ & 70.97 \\
\hline Planned pregnancy (yes) & 80.59 & $73.67 * * * *$ & $67.02 * * *$ \\
\hline
\end{tabular}

Two-tailed ANOVA and Chi square probability testing comparing the 'no binge' group with the 'early' and 'late' binge groups, separately

* Sig. at 0.05 ; ** sig. at 0.01 ; *** sig. at 0.001 ; **** sig. at 0.0001

a Tenant, homeless or living with parents

b Appears in the Psychiatric Central Registry before the age of seven of the child

c Summing the total number of reported drinks from the three interviews to obtain the total number of drinks during the entire pregnancy, excluding binge episodes

${ }^{\mathrm{d}}$ Pre-pregnancy body mass index within the normal range of 18.5 to $<25$

pregnancy [relative change in mean 1.21 (CI 1.04-1.42)]. Further when the early and late exposure groups are compared statistically, the difference is significant (results not shown here). For the above cutoff clinical scales, no statistically significant associations were found and the point estimates did not indicate any strong association (Table 3).

In the main analyses the early and late binge groups were compared to the no binge group irrespective of the number of binge episodes of each woman. To look at 
possible effects of the number of binge episodes, the analyses were replicated only including women with either one or at most two binge episodes. This left a total of 3,309 and 91 women in the early and late exposure groups, respectively. The results revealed virtually identical estimates for all outcomes. However, the OR estimates for the hyperactivity scores were a little lower compared to the main results. Furthermore, the sensitivity analyses including only women interviewed before 16 completed weeks also revealed virtually identical results (data not shown).

\section{Discussion}

No significant differences were observed in the main analyses looking at associations of binge drinking on any of the above clinical cutoff scale scores. However, since previous studies looking at prenatal exposure to binge drinking and associations with behavioural and emotional outcomes generally have only found weak associations, we did not a priori expect such associations [22, 23]. From this perspective it had not been expected that exposure to one or two binge episodes would lead to substantial childhood behavioural and emotional problems, i.e. a significant increase in the number of children with above clinical cutoff scores. However, it was expected that it would lead to an increase in mean score, i.e. lead to subtle behavioural differences.

The analyses did reveal significantly, elevated externalising estimates for children of early and late pregnancy

Table 2 Adjusted relative changes in means for the log-transformed externalising/internalising scores

\begin{tabular}{|c|c|c|c|}
\hline & $\begin{array}{l}\text { No binge } \\
(N=25,781) \\
\text { Relative } \\
\text { changes in } \\
\text { means }\end{array}$ & $\begin{array}{l}\text { Early binge } \\
(N=3,654) \\
\text { Relative } \\
\text { changes in } \\
\text { means }\end{array}$ & $\begin{array}{l}\text { Late binge } \\
(N=94) \\
\text { Relative } \\
\text { changes in } \\
\text { means }\end{array}$ \\
\hline Externalising & Ref. & $1.02(1.00-1.05)$ & $1.21(1.04-1.42)$ \\
\hline Internalising & Ref. & $1.00(0.98-1.03)$ & $1.05(0.89-1.24)$ \\
\hline
\end{tabular}

The analyses are adjusted for the following confounders: maternal education, psychiatric diagnoses, age and smoking binge drinkers, estimates that were stable even after excluding women with more than two binge episodes. In other words, being exposed to just one or two binge drinking episodes early or late in pregnancy was associated with an increased risk of subtle behavioural differences at age seven. However, the estimates were much higher for the late bingers compared to the early bingers. One systematic review looking at human binge studies concluded that there was a possible, but generally quite small, effect of exposure to binge drinking on neurodevelopmental outcomes [23]. The studies reported in the review all investigated exposure to early pregnancy binge drinking only, with results that are in line with the findings from the present study, where very weak associations were observed with early pregnancy exposure. Another study including women with binge episodes throughout pregnancy found similar weak associations with binge drinking, and only for boys [22]. However, as is apparent from the present study far fewer women report binge episodes in the last part of pregnancy compared to early pregnancy, potentially masking stronger effects of late exposure. The much lower group size here results in less reliable estimates and lower statistical power. To our knowledge no other humans studies have to date investigated the specific associations of being exposed to binge drinking exclusively in late pregnancy, and the results indicate that late exposure is actually associated with significantly more externalising problems at age seven compared to the group exposed in early pregnancy.

Previous studies have typically used data on maternal binge drinking limited to the early period of pregnancy and have most often found no or at most modest associations with subsequent development in the child $[24,25]$. The present findings suggest that it actually is important also to examine binge drinking late in pregnancy.

An obvious limitation of the present study is that the late binge group may consist of a selected group of women, since it appears from Table 1 that they are significantly less well educated, are more likely to smoke and to have a minimum of one psychiatric diagnosis. The generalizability of the results may therefore be limited. Also, parent ratings were used as the outcome measure in the study, although

Table 3 Adjusted ORs for the above cutoff hyperactivity, conduct, peer problems and emotional scores

\begin{tabular}{|c|c|c|c|c|c|c|}
\hline & \multicolumn{2}{|c|}{ No binge $(N=25,781)$} & \multicolumn{2}{|c|}{ Early binge $(N=3,654)$} & \multicolumn{2}{|c|}{ Late binge $(N=94)$} \\
\hline & Cases $(\%)$ & & Cases $(\%)$ & OR & Cases $(\%)$ & OR \\
\hline Hyperactivity & $4,748(18.42)$ & Ref. & $738(20.20)$ & $0.93(0.85-1.02)$ & $19(20.21)$ & $0.98(0.60-1.68)$ \\
\hline Conduct & $3,596(13.95)$ & Ref. & $521(14.26)$ & $1.01(0.91-1.11)$ & $17(18.09)$ & $0.81(0.49-1.43)$ \\
\hline Emotional & $3,401(13.19)$ & Ref. & $530(14.50)$ & $0.93(0.84-1.03)$ & $15(15.96)$ & $0.86(0.51-1.57)$ \\
\hline Peer problems & $5,019(19.47)$ & Ref. & $690(18.88)$ & $1.04(0.96-1.14)$ & $17(18.09)$ & $1.17(0.70-2.05)$ \\
\hline
\end{tabular}

The analyses are adjusted for the following confounders: maternal education, psychiatric diagnoses, age and smoking 
teacher ratings might have provided a more valid assessment of the behaviour of children. Binge drinking was defined as a minimum of five alcohol-containing units on a single occasion. In other studies binge drinking is often defined as four or more units. Further, a standard Danish drink is defined as $12 \mathrm{~g}$ of absolute alcohol, which is for example $4.1 \mathrm{~g}$ higher than a standard UK drink. This means that the total exposure is higher and this could have affected the results. Therefore, researchers are encouraged to investigate whether the findings from our study can be replicated across other samples.

Acknowledgments The study was supported financially by the Department of Psychology, University of Copenhagen; Ludvig og Sara Elsass Foundation; Aase og Ejnar Danielsens Foundation; Carl J. Becker's Foundation; the Lundbeck Foundation; Børne- og Ungdomspsykiatrisk Selskab i Danmark; Dagmar Marshalls Foundation; The A.P. Møller Foundation for the Advancement of Medical Science; and Direktør Jakob Madsens Legat.

Conflict of interest There are no declared conflict of interests for any of the authors.

\section{References}

1. Irner TB (2012) Substance exposure in utero and developmental consequences in adolescence: a systematic review. Child Neuropsychol 18(6):521-549

2. Gray R, Henderson J (2007) Review of the fetal effects of prenatal alcohol exposure. University of Oxford, National Perinatal Epidemiology Unit

3. O'Leary CM, Zubrick SR, Taylor CL, Dixon G, Bower C (2009) Prenatal alcohol exposure and language delay in 2-year-old children: the importance of dose and timing on risk. Pediatrics 123(2):547-554

4. Willford J, Leech S, Day N (2006) Moderate prenatal alcohol exposure and cognitive status of children at age 10. Alcohol Clin Exp Res 30(6): 1051-1059

5. Alvik A, Aalen OO, Lindemann R (2013) Early fetal binge alcohol exposure predicts high behavioral symptom scores in 5.5-year-old children. Alcohol Clin Exp Res 37(11):1954-1962

6. Andersen SL (2003) Trajectories of brain development: point of vulnerability or window of opportunity? Neurosci Biobehav Rev 27(1-2):3-18

7. Rice D, Barone S Jr (2000) Critical periods of vulnerability for the developing nervous system: evidence from humans and animal models. Environ Health Perspect 108(Suppl 3):511-533

8. Schlotz W, Phillips DI (2009) Fetal origins of mental health: evidence and mechanisms. Brain Behav Immun 23(7):905-916

9. Kesmodel U (2001) Binge drinking in pregnancy-frequency and methodology. Am J Epidemiol 154(8):777-782
10. Abel EL (1998) Neurodevelopmental abnormalities. Behavioural and cognitive. Fetal alcohol abuse syndrome. Plenum, New York, pp 111-138

11. Schneider ML, Moore CF, Becker EF (2001) Timing of moderate alcohol exposure during pregnancy and neonatal outcome in rhesus monkeys (Macaca mulatta). Alcohol Clin Exp Res 25(8):1238-1245

12. Maier SE, Miller JA, Blackwell JM, West JR (1999) Fetal alcohol exposure and temporal vulnerability: regional differences in cell loss as a function of the timing of binge-like alcohol exposure during brain development. Alcohol Clin Exp Res 23(4):726-734

13. Mooney SM, Varlinskaya EI (2011) Acute prenatal exposure to ethanol and social behavior: effects of age, sex, and timing of exposure. Behav Brain Res 216(1):358-364

14. Olsen J, Melbye M, Olsen SF, Sorensen TI, Aaby P, Andersen AM et al (2001) The Danish National Birth Cohort-its background, structure and aim. Scand J Public Health 29(4):300-307

15. (2013) Danish National Birth Cohorts: the interviews. Statens Serum Institut. http://www.dnbc.dk

16. Goodman R (1997) The strengths and difficulties questionnaire: a research note. J Child Psychol Psychiatry 38(5):581-586

17. Goodman R, Scott S (1999) Comparing the strengths and difficulties questionnaire and the child behavior checklist: is small beautiful? J Abnorm Child Psychol 27(1):17-24

18. Goodman R (2001) Psychometric properties of the strengths and difficulties questionnaire. J Am Acad Child Adolesc Psychiatry 40(11):1337-1345

19. Niclasen J, Skovgaard AM, Andersen AM, Somhovd MJ, Obel C (2013) A confirmatory approach to examining the factor structure of the Strengths and Difficulties Questionnaire (SDQ): a large scale cohort study. J Abnorm Child Psychol 41(3):355-365

20. Niclasen J (2012) SDQ Danish cut off scores: 5-7-year-olds. http://www.sdqinfo.com/DanishNorms/DanishNorms.html

21. Niclasen J, Teasdale TW, Andersen AM, Skovgaard AM, Elberling H, Obel C (2012) Psychometric properties of the Danish Strength and Difficulties Questionnaire: the SDQ assessed for more than 70,000 raters in four different cohorts. PLoS ONE 7(2):e32025

22. Niclasen J, Andersen AM, Teasdale TW, Strandberg-Larsen K (2013) Binge drinking and cumulated alcohol exposure in pregnancy: behavioural and emotional development at age seven. J Epidemiol Community Health (2013). Published Online First. doi:10.1136/jech-2013-202956

23. Henderson J, Kesmodel U, Gray R (2007) Systematic review of the fetal effects of prenatal binge drinking. J Epidemiol Community Health 61:1069-1073

24. Kesmodel US, Eriksen HL, Underbjerg M, Kilburn TR, Stovring H, Wimberley $\mathrm{T}$ et al (2012) The effect of alcohol binge drinking in early pregnancy on general intelligence in children. BJOG 119(10):1222-1231

25. Sayal K, Heron J, Golding J, Alati R, Smith GD, Gray R et al (2009) Binge pattern of alcohol consumption during pregnancy and childhood mental health outcomes: longitudinal populationbased study. Pediatrics 123(2):e289-e296 